\title{
A Multimedia Medical Communication Link Between a Radiology Department and an Emergency Department
}

\author{
M. Goldberg, J. Robertson, G. Bélanger, N. Georganas, J. Mastronardi, S. Cohn-Sfetcu, \\ R. Dillon, and J. Tombaugh
}

\begin{abstract}
The most critical aspect of a radiologist's work is communicating his findings to the attending physician responsible for the patient's care. This is also the part of the process that is least well organized and the most subject to failure. At the University of Ottawa Medical Communications Research Centre we are investigating technical means to improving communications between radiologists and attending physicians. We first introduce the radiology communication service problem and show why it is essentially a multimedia communication problem. We then briefly describe a multimedia communication system designed and implemented by our research team. The multimedia system consists of several work stations linked by the Hospital's local area network (LAN). Each physician work station comprises a Compaq 386 / 20-Mhz microcomputer with 16 Mbytes of RAM, a 500-Mbyte image disk, and an image memory that drives a 1,000-line monochrome monitor. The images are digitized using a Konica laserbased film digitizer (2430 by 2000 10-bit pixels for a standard chest radiograph). The multimedia file server manager station is built around a PC-AT compatible with a Northern Telecom Meridian SL1ST digital PBX and a Meridian Mail digital voice messaging system. This last device is used to store voice data and is linked via the PBX to the workstations' digital telephones. A Sytek 6,000 LAN links all work stations to the file server. All data, image, and graphic information is transmitted via this network, while the twisted pair connections linking the digital PBX to the telephone sets are used for transmitting voice data. Finally, we provide details of an inhospital trial linking the Department of Radiological Sciences and the Emergency Department at the Ottawa Civic Hospital, a 950-bed tertiary care teaching hospital.
\end{abstract}

(C) 1989 by W.B. Saunders Company.

From the Telecommunications Research Institute of Ontario, University of Ottawa Medical Communications Research Centre, Department of Electrical Engineering; Ottawa Civic Hospital, Department of Radiological Sciences; Bell-Northern Research, Ltd, Ottawa; and Carleton University, Department of Psychology, Ottawa.

Address reprint requests to Telecommunications $\boldsymbol{R e}$ search Institute of Ontario, University of Ottawa Medical Communications Research Centre, Department of Electrical Engineering, 161 Louis Pasteur, Ottawa, Ontario K1N6N5, Canada.

(C) 1989 by W.B. Saunders Company.

0897-1889/89/0202-0007\$03.00/0
N IMPORTANT facet of medical practice $A$ is the consultative process that occurs between specialists and the attending physician. During this consultation, information about the patient is exchanged and opinions are given to the attending physician. This consultative process can occur through a face-to-face meeting, a telephone conversation, or a series of written messages passed between physicians. This process is in general time-consuming, inefficient, and can cause delays in patient treatment. In all cases the onus is still on the attending physician to collect and provide the relevant patient files. We refer to teleconsultation as the process of carrying out a consultation at a distance through telecommunication facilities. Teleconsultation, as we envisage the system, should offer the physicians simultaneous, shared access to the patient file, which they can manipulate and annotate. We are particularly interested in the teleconsultation process as it relates to radiology, initially for intrahospital communication and, eventually, for interhospital communication.

Patient files contain a large number of data sets of different types and formats, including handwritten requests, typed forms, computerbased data, voice messages/reports, and diagnostic information such as ECGs and radiographic images. By far the largest component of the file is the diagnostic imagery. We note that the patient files are really multimedia documents, and, therefore, require the use of multimedia communication and storage technologies. These multimedia documents containing voice, image, and text segments need to be shared during a consultation session between an attending physician and a radiologist. The rapid communication of diagnostic information together with the patient files will lead to improved patient care and can reduce the length of time spent in the hospital by the patient. Both factors are important incentives for the introduction of a multimedia communication system.

Research in radiology communication is presently focused on the problems of image transmis- 
sion and storage of diagnostic images, ${ }^{1}$ teleradiology for remote sites, and picture archiving and communications systems (PACS) for in-hospital use. In teleradiology, the images are acquired and digitized at some remote site and then typically transmitted over a low-data rate telephone line to a centralized radiology service. In the long run, all PACS images will be acquired in digital form and stored in a large image database. The images would then be distributed through some form of local area network (LAN) for physician viewing on work stations. In PACS or the so-called total digital radiology solution, all images would be stored, transmitted and read in digital format. There are a number of different facets to this research.

1. Image storage and management ${ }^{2}$ : There are many design issues that include the physical media to use, optimization of storage and access, integrity, performance.

2. Communications: The main concerns are LAN architectures, transmission protocols, and performance issues. In terms of LANs the options range from existing systems such as Ethernet and extend to high-speed photonic networks. ${ }^{3}$ An excellent overview of the state of the art is provided by Noz et al. ${ }^{4}$ In terms of protocols, interest focuses on the American College of Radiology and National Electrical Manufacturers Association (ACR-NEMA) standards for medical digital image data interchange. However, this standard does not address the integrated multimedia data problem. ${ }^{5}$ Performance studies concern the measurements of traffic patterns and related simulations with emphasis on large centralized image archives.,

3. Terminal/work station: There is interest in replacing the ubiquitous viewing light box with its digital equivalent. ${ }^{8}$ Research is concentrated on the spatial/spectral resolution requirements, ${ }^{9}$ user interfaces, ${ }^{10-12}$ and data organization and display. ${ }^{13}$ This research area is being closely monitored, as it has direct relevance to our project.

4. System (component) integration ${ }^{14,15}$ : There is a wide variety of system components available for building a PACS system. A number of research efforts are directed towards building prototype systems for system evaluation, including the problem of integration with hospital information services. ${ }^{16}$
The main shortcoming of this research is that it does not address the need for physicianradiologist consultation on the multimedia data.

In this article we describe a multimedia radiology communication system we have implemented, which will be undergoing clinical inhospital evaluation. This system will link the Departments of Radiology and Emergency of the Ottawa Civic Hospital, a large tertiary care teaching hospital. Images acquired in Emergency will be digitized and made available on digital work stations, and a digital voice storage device will be used for voice messaging and reporting. Communication software will provide integrated access to the multimedia patient files and provide a real-time conferencing facility on work stations located in the two departments.

In the following sections, we describe in more detail the radiology communication problem and demonstrate that it involves multiple media; we present the multimedia radiology communication system and show how it can address some of the radiology communication problems; we briefly review the areas of research required to support our scenario; and we provide details of the in-hospital trials.

\section{THE RADIOLOGY COMMUNICATION PROCESS}

Radiographs are one of the most important sources of diagnostic information. The steps involved from the actual request for a radiographic examination by the attending physician to his obtaining the radiologist's report are outlined in Fig 1. From this diagram it can be appreciated that this information flow involves considerable movement of people, paper, and $X$-ray film from place to place with all of the attendant opportunities for delay and loss. Following the path of the typical radiological in-patient examination we find the following.

1. Ordering: On morning rounds, the physician orders a radiological examination for a patient by filling out a form with spaces for the type of examination and whatever clinical information the physician decides to include. This form is sent by intrahospital mail to the radiology department where the examination is scheduled.

2. Acquisition: The patient is taken to radiology where the examination is performed. Once 


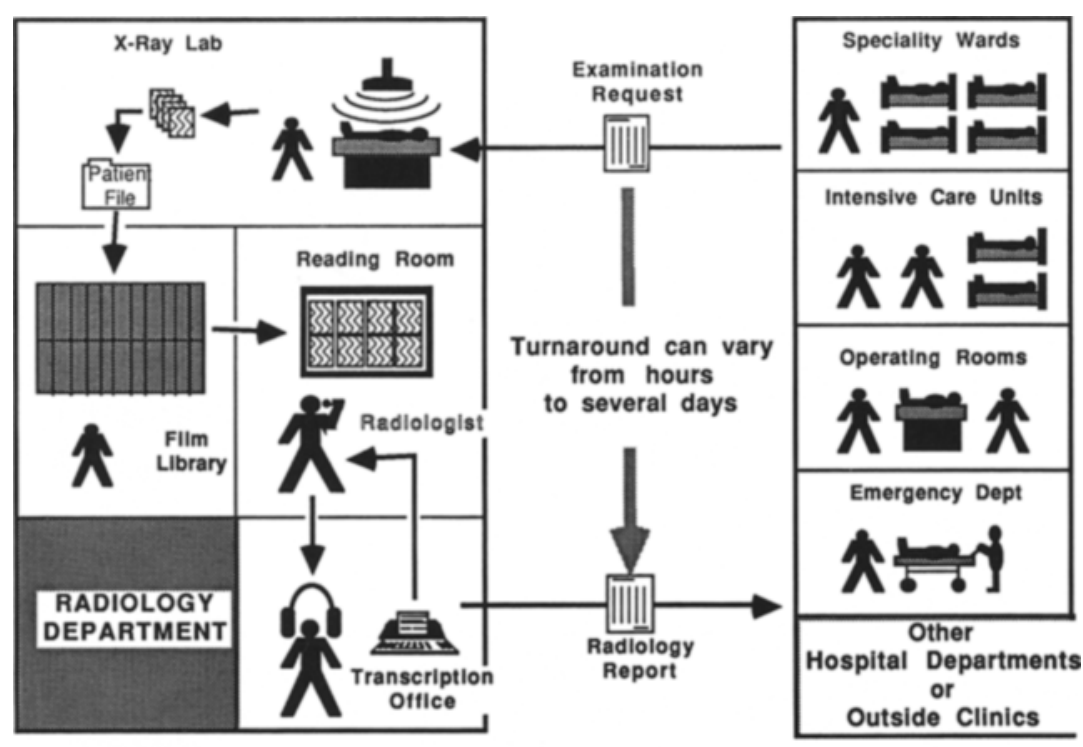

Fig 1. Radiology communication service problem. the films are developed, they are sent to the film library where they are logged and collated with the patient's existing radiology file. The patient's entire radiology file is then added to the batch of files that will be sent to a particular radiologist for interpretation and reporting.

3. Reading: The radiologist reads the cases and reports them by dictating an opinion into a dictating machine. At some point in the day the dictation cassettes are picked up and delivered to the transcribing pool. Here the reports are transcribed and then returned to the radiologist in batches for verification and signature. The signed reports are returned to the transcription pool for distribution to the film library, the primary care physicians, and other locations. On average it takes from three to five days for the radiologist's report to reach the patient's chart from the time a primary care physician orders a radiological examination.

4. Consultation: If the primary care physician needs or wants the results of the radiographic examination before the report reaches the patient's chart, he can find and interpret the images himself, find the images and a radiologist and have the radiologist interpret the images, or telephone the radiology department and, if the radiologist's report has been transcribed, have the clerk read it to him.

The actual acquisition of the radiographs is only one step in the process. Most important, from the viewpoint of patient care, are the following: the referral process, in which the attending physician requests an examination in order to confirm some clinical suspicion; the reporting process, in which the radiologist reads the films and reports his findings; the consultation process, in which the radiologist and one or more attending physicians review the case and more clinical information is shared; and the film management process, in which the patient's films are properly stored for future reference.

A number of points regarding the process need to be highlighted. The most important point is the length of time that is required from the first to last step. For in-hospital patients the normal turnaround time is one to three days, whereas for out-patients this delay can take more than a week. An important source of these delays is the need to manually manipulate the patient files. These delays can decrease the quality of patient care, increase the length of patient hospital stay, and often are the root cause of extra diagnostic procedures being performed. Therefore, there is a significant impetus from the medical community to improve the processing of radiographs. A second observation is that radiologists are specialists trained to read radiographic images and explain the results to attending physicians. A written report may suffice, but frequently the physician needs to see the radiographs with the report, or to directly consult the radiologist. Typically, the attending physician will have clinical information that changes the radiologist's 
interpretation. In other situations the findings of the radiologist may not be apparent (readily visible) to the attending physician. To arrange a consultation, the attending physician normally spends more than 15 minutes gathering the films and finding a radiologist for a one-minute consultation. A third point is that a patient film file is accessed on average 14 times during a hospital stay, leading to a serious problem in keeping track of the films; about $10 \%$ to $20 \%$ of the time the films are not available and about $1 \%$ they are permanently lost or misplaced. The overall conclusion that we can draw is that radiology communication does not simply involve the transfer of images, but also includes a number of other data sets that must be managed and manipulated in an integrated and coordinated fashion.

\section{MULTIMEDIA RADIOLOGY COMMUNICATION SYSTEM}

Figure 2 shows a diagram of the multimedia radiology communication system developed at the University of Ottawa that is being installed at the Ottawa Civic Hospital. A detailed description of the design and architecture of the system has been presented. ${ }^{17}$

The system contains four nodes: a file server and three work stations, namely, radiologist, physician, and image entry work stations. The radiologist and physician work stations comprise Compaq 386/20 computers, and contain an image memory card that drives a 1,000-line monochrome monitor, a 500-Mbyte image disk, a communications interface card, and a digital telephone. The image entry work station is similar to the physician and radiologist work stations, with the addition of a Konica radiographic digitizer (yielding 2,430 by 2,000 10-bit pixel images for standard chest radiographs) and a larger 800-Mbyte image disk. The multimedia file server manager station is built around a PC-AT compatible with a Northern Telecom Meridian SL-1ST digital PBX and a Meridian Mail digital voice messaging system. This last device is used to store voice data and is linked via the PBX to the work stations' digital telephones.

A Sytek 6000 LAN links all work stations to the file server. All information is transmitted via this network, while the twisted pair connections linking the digital PBX to the telephone sets are used for transmitting voice data. The choice to

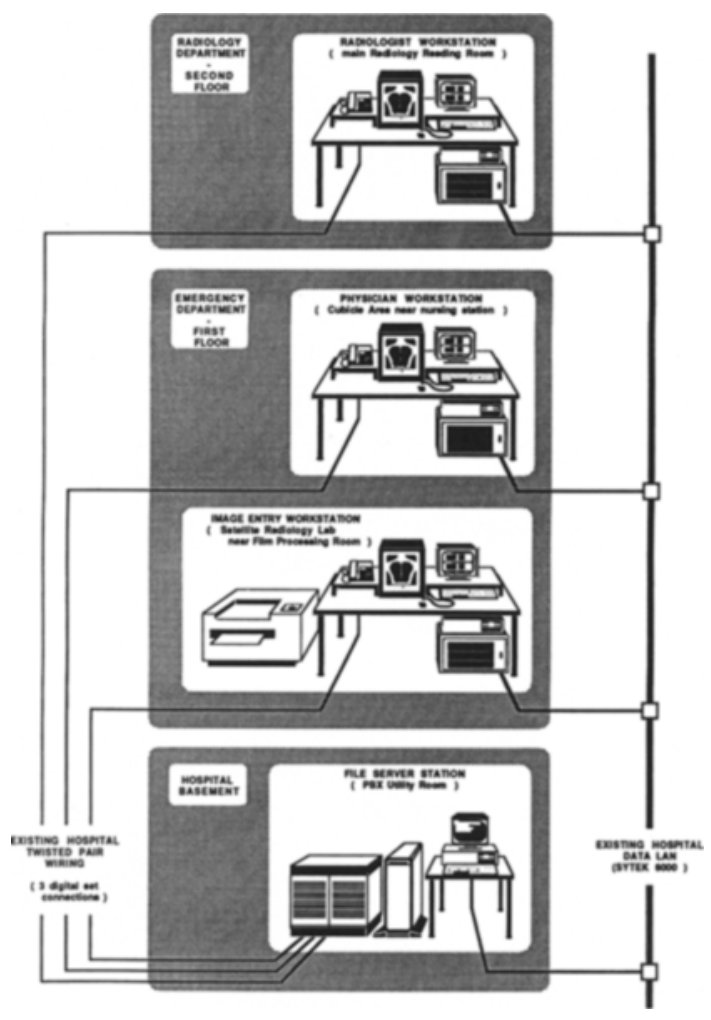

Fig 2. Ottawa Civic Hospital Emergency/Radiology trial.

use a separate transmission support for voice was made, considering that most hospitals have a well-established telephone system based on PBX and twisted pair connections.

The distribution of image data, by far the largest component of the patient files, is an important consideration. Radiological images are large; for standard chest radiographs, about 40 Megabits. To provide for the rapid display of the images and to reduce network traffic, the system stores a subsampled version of the image ( 8 Megabits) in the workstation's hard disk, while keeping the full resolution version in the image entry workstation store. This strategy allows the physician to rapidly access the images in a patient file from the local image disk. The physician always has the option of magnifying any area of interest, in which case the data are obtained from the central image server over the network. The system also provides automated image enhancement facilities, as well as various simple image manipulation functions such as mensuration and cropping.

The distributed system architecture furnishes 
the base for providing the creation of a shared visual workspace (the image monitor and the computer monitor) and the use of a voice channel. A radiologist and a physician are thus able, working at their own workstation, in their own department, to engage in a consultation while sharing the entire patient file.

Our studies indicate that the application of multimedia communication can help the Department of Radiology provide better services to the attending physicians in the following ways.

1. Remote access: by simply allowing the attending physician remote electronic access to the radiographs and dictated reports, waiting times can be reduced from days to minutes.

2. Shared visual work space: the physicianradiologist consultation process will also be facilitated through the creation of a shared visual work space on two remotely located work stations. In certain critical situations such as emergency, intensive care, or surgery this shared visual workspace facility could improve patient care. In almost all other cases it will make more efficient use of the physician's time.

3. Multimedia patient files: by linking the voice messages and reports with the images and other patient data the attending physician will have a more complete patient file available.

We note the following changes in the path of an in-patient examination after a multimedia radiological communications system has been installed (Fig 3).

1. Ordering: the physician orders a radiological examination via the multimedia communica- tions system. He can also leave voice messages for the radiology staff concerning the patient and consult with either the technicians or a radiologist to determine the optimum type of examination, etc.

2. Acquisition: the patient is taken to radiology and the examination performed. Once the films are developed they are digitized and automatically collated with the patient's chart. At this point the images are available to the physician via his work station. The system assigns the case to a radiologist and puts it into his message queue, with a priority code depending on the patient's condition.

3. Reading: the radiologist, as before, reads cases as time permits. However, he now has the patient's detailed medical history at hand and possibly additional information in the form of a voice message from the clinician. The radiologist can prepare multimedia reports so that features of interest in the images are viewed during the report. Since the radiologist dictates his report into the system it is available to both the transcription pool and the primary care physician as soon as the radiologist has finished the report.

4. Consultation: this takes place through the system, either asynchronously by leaving voice messages or synchronously using the shared visual work space. In either case they will make use of image, text, and voice data simultaneously.

\section{IN-HOSPITAL TRIALS}

Our first hospital trials entailed installing a multimedia communication system between the

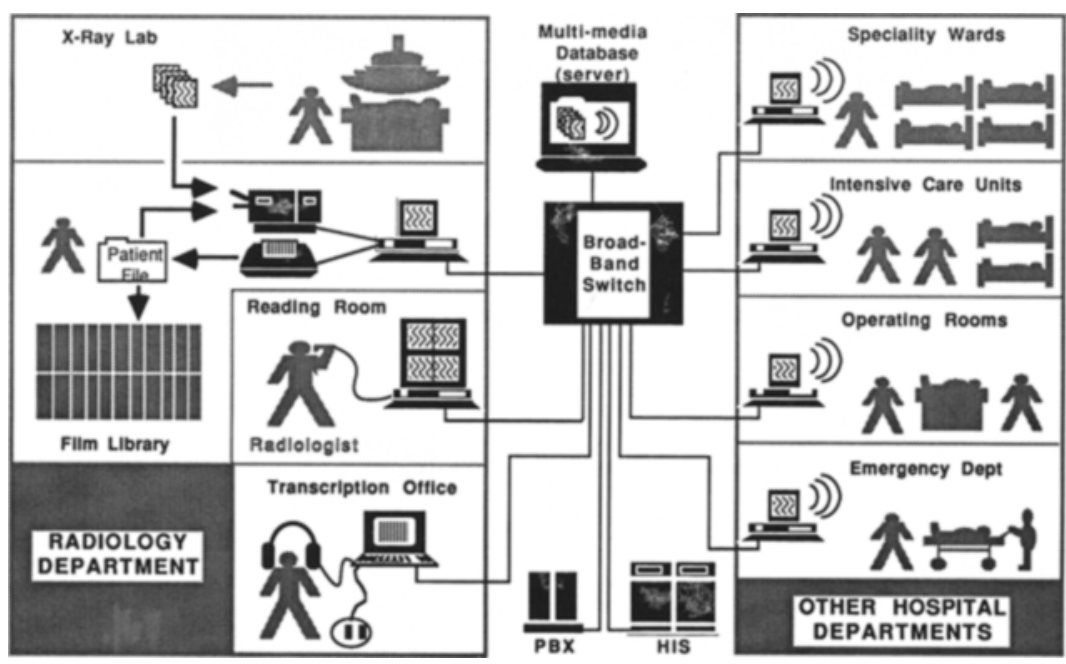

Fig 3. Multimedia Radiology communication solution. 
Emergency and Radiology Departments at the Ottawa Civic Hospital. The advantages of theEmergency department as a first trial site are: patients stay a maximum of 24 hours in Emergency, eliminating the need to develop an extensive image and data management and storage capability; emergency patients present the widest possible range of pathologies and conditions, thus providing a complete range of radiographic examinations; and the range of radiological interpretative skills range from poor (interns) to excellent (emergentologists).

\section{Protocol}

During the trials the experimental protocol is as follows: (1) The emergentologist orders a radiographic examination as usual. The requisition is scanned and added to the patient's multimedia file. The emergentologist may also attach a voice message to the patient's file if need be; (2) once the films have been developed, they are digitized and distributed to the radiologist's and emergentologist's work stations. The radiologist is alerted by the system that there is a case waiting for attention. At the same time a runner delivers the original films to the radiologist; (3) the radiologist first views the digital images and dictates a report based on the digital image, then examines the analog image (film). If some change is required to the report after viewing the analog image, then a new report is dictated. An observer debriefs the radiologist immediately to determine the reason for the change. This protocol will satisfy medical-legal requirements, help train radiologists to develop techniques to view digital images, and provide data for use in developing image enhancements; (4) as soon as the radiologist finishes with the case the system alerts the emergentologist, who receives the films and makes the same comparison as the radiologist, with the benefit of the radiologist's report. At any time the radiologist and the emergentologist can use the system to consult with each other or to pass along voice messages.

During the trial period we are conducting investigations into four broad areas: system utility, medical performance, technical performance and usability. Under utility we are assessing the value of multimedia files and a shared visual work space to radiologists and clinicians. For example, how often is the facility used? for what cases is it used? what is the clinical value? Medical performance testing encompasses measuring diagnostic performance of the images and testing various automatic image enhancement techniques. The main question we ask is: how do reports differ between reading from the analog film and the digital image on the monitor? Under technical performance we are measuring the effectiveness of using a distributed image database in terms of communication system delays for image transfer and distribution, and at the work station level, the performance in terms of response times to user requests.

We have divided the usability studies into four areas. (1) Ease of learning: how quickly do the physicians learn to use the system, both for occasional and frequent users? (2) Changing usage patterns: does the usage of the system change with time? (3) Ease of use: how easy is the workstation to use in terms of the userinterface commands? (4) Interface consistency: is the interface perceived to be consistent with respect to its structure and syntax?

The details of the methodology for the usability studies and the evaluation of the work stations have been reported. ${ }^{18-28}$

\section{PROJECT PARTICIPANTS}

The multimedia radiological communication system is the result of a large multidiscipline, multi-institution research and development effort. In Fig 4 we show in summary form the areas of expertise of the four partners. The industrial partner Bell-Northern Research, Ltd (Ottawa) was responsible for the technological issues and contributed hardware and engineering manpower to the joint project. The University of Ottawa Department of Electrical Engineering

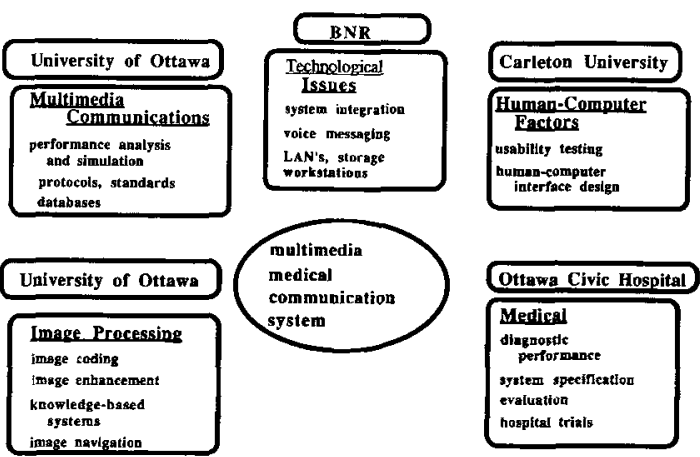

Fig 4. Expertise of project participants. 
undertook much of the early research and was responsible for its implementation in the system. The Carleton University Department of Psychology developed the user testing methodology and helped design the user interface. The Departments of Radiology and Emergency of the Ottawa Civic Hospital provided the initial system specifications and participated in the extensive preliminary and final testing of the multimedia radiology communication system.

\section{CONCLUSIONS}

In this article we first show that radiology communication is essentially multimedia in nature. We then show how a multimedia communi- cations system can improve radiology communication, specifically, transfer of images, messaging, reporting and consultation. We then briefly describe a multimedia communication system installed at the Ottawa Civic Hospital and which will be used to link the Departments of Radiology and Emergency. The objectives and protocols of the on-going hospital trial are detailed.

\section{ACKNOWLEDGMENT}

The authors wish to thank the radiologists, emergentologists, and personnel of the Ottawa Civic Hospital, who generously donated their time to our experiments. This work was funded by the Telecommunications Research Institute of Ontario (TRIO), Bell Canada, and Bell-Northern Research (BNR).

\section{REFERENCES}

1. Schneider RH, Dwyer SJ III: Third International Conference on PACS for Medical Application. SPIE 536, 1985; Application of optical instrumentation in medicine and PACS. SPIE 626, 1986; Medical imaging. SPIE 767, 1987

2. Rutherford HG, Gray M, Harvey J, et al: Highspeed multimodality digital image management system. SPIE 454: 103,1984

3. Arenson RL, et al: Early experiences with fiber optics PACS for medical images. SPIE 418:117, 1983

4. Noz ME, et al: Local area networks in an imaging environment. Crit Rev Med Informatics 1:81-153, 1986

5. National Electrical Manufacturers Association: ACRNEMA Digital Imaging and Communications Standard. Washington, DC, National Electrical Manufacturers Association, 1985, p 192

6. Bijl K, et al: Radiological workload information as a prerequisite for the configuration of a medical image archive and communication network. SPIE 626:717.722, 1986

7. Lawrence GR, et al: Hospital PACS network simulation studies. SPIE 626:729-739, 1986

8. Rutherford HG, Gray MJ: Digital lightbox, one of the integral pieces of PACS. SPIE 418:60, 1983

9. Seeley GW, Newell JD: The use of psychophysical principles in the design of a total digital radiology department. Radiol Clin North Am 23:341-355, 1985

10. Massicotte JB, Wurtz RE, Benster RW, et al: A menu driven interface for a physician's imaging console. SPIE 536:158-164, 1985

11. Wendler $\mathrm{T}$ : Human-Computer Interaction in Radiology, Proceedings of Computer Assisted Radiology, Berlin, 1985

12. van der Voorde $F$, Arenson $R$, Kundel $H$, et al: Development of a physician-friendly digital image display console. SPIE 626:541-548, 1986

13. Kasday LR: Human factors consideration in PACS design. SPIE 626:581-592, 1986

14. Hemminger $B M$, et al: The ongoing implementation of a prototype medical communication system at the University of North Carolina. SPIE 626:574-580, 1986

15. Mun SK, Choyke A, Duernick X, et al: Development of PACS at Georgetown University Radiology Department. SPIE 536:229-236, 1985

16. de Valk J, Bijl K, Bakker AR, et al: The Dutch imagis project. Proc Image Sci 72-78, 1985

17. Mastronardi J, Reed S, Goldberg M, et al: Design of a multimedia communication system for clinical trials at the Ottawa Civic Hospital. SPIE 1093, 1989

18. Tombaugh JW, Dillon RF, Coristine M: Goal setting and user testing to ensure a PACS interface satisfactory to radiologists. SPIE 1093, 1989

19. Bélanger G, Robertson JG, Corisitne M, et al: Evaluation of workstation by clinicians. SPIE 1093, 1989

20. Schramm C, Goldberg M: Multimedia radiological reports: Creation and playback. SPIE 1091, 1989

21. Roger E, Goldberg M: Image organization and navigation strategies for a radiological workstation. SPIE 1091, 1989

22. Carboni N, Tombaugh JW, Dillon RF: The effect of simultaneous vs sequential viewing of digital images on comparative judgement performance. SPIE 1091, 1989

23. Dillon RF, Goldberg M, Roger E, et al: User testing of the image navigation system for a radiological workstation. SPIE 1091, 1989

24. Panchanathan S, Goldberg M: A mini-max error criterion based algorithm for image adaptive vector quantization. SPIE 1091, 1989

25. Plessis B, Goldberg M, Dillon R, et al: Contextdependent enhancements for radiological images. SPIE 1092, 1989

26. Karmouch A, Georganas ND: Multimedia document architecture for medical applications. SPIE 1093, 1989

27. Vallée R, Orozco-Barbosa L, Georganas ND: Modelling and simulation of multimedia communication networks. SPIE 1093, 1989

28. Coristine M, Tombaugh JW, Dillon RF: Observational assessment of field-trial site for the implementation of a PACS network. SPIE 1093, 1989 